\title{
Mobilum - a new mobile app to engage visuospatial processing for the reduction of intrusive visual memories
}

\author{
Henrik Kessler $^{1 \#}$, Luisa Dangellia ${ }^{1 \#}$, Ralf Kessler ${ }^{2}$, Vincent Mahnke ${ }^{3}$, Stephan Herpertz ${ }^{1}$, Aram Kehyayan ${ }^{1}$ \\ ${ }^{1}$ Department of Psychosomatic Medicine and Psychotherapy, LWL-University Hospital, Ruhr University Bochum, Bochum, Germany; ${ }^{2}$ DAEDALIC \\ Entertainment GmbH, Hamburg, Germany; ${ }^{3}$ Sunlab GmbH, Aschaffenburg, Germany \\ \#These authors contributed equally to this work. \\ Correspondence to: Henrik Kessler. Department of Psychosomatic Medicine and Psychotherapy, LWL-University Hospital, Ruhr University Bochum, \\ Alexandrinenstrasse 1-3, 44791 Bochum, Germany. Email: henrik.kessler@ruhr-uni-bochum.de.
}

\begin{abstract}
Intrusive memories are a key symptom of posttraumatic stress disorder (PTSD), a prevalent condition causing considerable personal suffering, and entailing large direct and indirect societal costs. While effective treatment options for PTSD exist, on a global scale they are not readily available to many patients in need. In the last years, several studies have shown that the computer game Tetris can reduce the frequency of intrusive memories in healthy subjects (after a trauma analogue), in populations at high risk of developing PTSD, as well as in patients already suffering from PTSD. The presumed mechanism behind this effect is that both Tetris and intrusions require-and therefore compete for-limited visuospatial working memory resources. In search for a new alternative tool that can engage visuospatial processing as effectively as Tetris, we developed a game named Mobilum. This is an app for Android devices, in which users have to rotate in an imaginative way around a translucent virtual cube in order to decide from which perspective a complex three-dimensional figure inside the cube is seen. Mobilum was developed to investigate whether the intrusion-reducing effect is exclusively inherent to Tetris, or if it can be achieved with another task engaging users in visuospatial processing. Also, unlike available versions of Tetris, Mobilum offers full control over key game parameters (e.g., difficulty, game duration), and is free of copyright and commercial issues. In this method paper, we describe the new Mobilum app, its theoretical background and development. Additionally, the first data on usability and feasibility are reported, as rated by $\mathrm{N}=16$ inpatients. Finally, we will provide an outlook on the next steps necessary to investigate Mobilum's potential as a therapeutic tool.
\end{abstract}

Keywords: Visuospatial task; intrusive memories; posttraumatic stress disorder (PTSD); Mobilum

Received: 01 September 2019; Accepted: 19 September 2019; Published: 22 November 2019.

doi: $10.21037 /$ mhealth.2019.09.15

View this article at: http://dx.doi.org/10.21037/mhealth.2019.09.15

\section{Introduction}

Potentially traumatic events occur often (1), and a fraction of those people affected eventually develop posttraumatic stress disorder (PTSD). With lifetime prevalences ranging between $1.3 \%$ (Japan) and 8.8\% (Northern Ireland), PTSD is of high epidemiological relevance (2). Only a small minority of patients actually receive adequate treatment, because existing effective treatments according to NICE- (3), AWMF- (4) and APA- (5) guidelines are not easily available on a global scale (6). This leads to suffering among patients and high direct and indirect societal costs (7). Hence there is a great need to develop and deploy novel treatment methods that can effectively mend PTSD symptoms, but are available and applicable on a much larger scale than existing treatment options (8). One approach to tackle this is the digitalization of treatment methods (in order to increase availability) and the translation of basic science knowledge to clinical applications (translational medicine) in order to specifically address key symptoms of PTSD (precision medicine). There are various ongoing developments in this vein with both healthy as well as clinical populations. 
Examples are computer programs to change dysfunctional cognitive bias in healthy participants (9-11) and PTSD patients (12), or to train proactive interference control $(13,14)$. Another very promising approach that we will further focus on is the idea to use visuospatial tasks in order to reduce the frequency of intrusive memories. Intrusions are memories, typically in the form of mental images, which occur involuntarily ("pop into one's mind"), cause suffering and are difficult to control. They can be short images or longer films of traumatic events and are a key symptom of PTSD $(15,16)$. The approach to target such intrusions with visuospatial tasks stems from the cognitive science concept of dual-task interference: Mental images or films are processed by visuospatial working memory components with limited capacities ("bottleneck"). This processing can hence be disturbed by a concurrent task exploiting those limited resources. Activating a mental image of a past (traumatic) event and performing a demanding visuospatial task at the same time leads to interference, which typically decreases vividness and emotionality of the mental image (17-21). This interference could also account for (part of) the effect of Eye Movement Desensitization and Reprocessing (EMDR) $(22,23)$. Very promising effects to reduce intrusions have been achieved with the well-known computer game Tetris. In this visuospatially demanding game, participants have to rotate blocks that fall down the screen in order to create continuous lines of blocks on the base of the screen. Emily Holmes and colleagues have repeatedly shown that Tetris can indeed reduce the frequency of intrusions in healthy participants when applied during or directly after watching a so-called trauma film (containing visually disturbing footage that typically causes intrusions in the aftermath) (24-27). First clinical studies with Tetris also show promising results: When played within 6 hours after a road traffic accident (28) or traumatic childbirth (29), Tetris effectivley decreased intrusions in the following week compared to control conditions. Recent studies with Tetris are additionally based on the concept of a reconsolidation-update: memories that have al ready been consolidated can still be changed later after having been reactivated and subjected to interference $(30,31)$. With this in mind, even traumatic memories that have been consolidated long ago should be susceptible to visuospatial interference if they were reactivated beforehand (32). Playing Tetris $24 \mathrm{~h}(33)$ or even $72 \mathrm{~h}(34,35)$ after a trauma film effectively reduced the frequency of intrusions in the following time period. A first clinical study in PTSD patients showed that recalling traumatic memories (writing them down) and playing Tetris afterwards can decrease the frequency of intrusions of precisely that particular memory which has been targeted by this intervention (36). Should those early results hold true in the future, visuospatial tasks could provide a widely available, efficient and easy-to-use method to mend a key symptom of PTSD, intrusive memories. In this method paper we present a new visuospatial task, Mobilum, that we developed as a mobile app to further explore the potential to affect intrusive visual memories.

\section{Rationale for developing Mobilum}

Previous research that has mainly used Tetris has repeatedly shown that visuospatial tasks performed shortly after a reminder of already consolidated visuospatial memories from a trauma film can disturb the reconsolidationupdate process (interference due to competition for the same limited visuospatial working memory resources) and lead to a reduction in intrusion frequency in the following days. We chose to critically extend previous research, including our own with Tetris, by introducing an alternative visuospatial task to Tetris. The rationale for developing a new task is as follows: first, the idea was to provide evidence for a mechanistic explanation that it is a visuospatially engaging task per se, rather than a specific game, that might interfere with intrusion processing. If future studies showed that Mobilum can reduce the frequency of intrusions at least as effectively as Tetris, this would be a point for general features of visuospatial tasks that might cause the effect, which is hence not confined to Tetris specifically. Second, as a methodological point, with our own game we have full control over many variables potentially modulating the effectiveness of the intervention: difficulty levels, duration of game play, levels of visuospatial engagement, and others. In contrast, when using Tetris the key game parameters are already provided by the software company and cannot be changed by researchers to be tailored for specific study designs. Finally, Tetris in its current form is a commercial mobile app developed for entertainment purposes by a large software company. If continuous research further establishes that Tetris as a specific game has health care implications by reliably mending a core symptom of PTSD, potential commercial or copyright issues might become important. This could conflict with the idea of free research and the vision to provide the new intervention on a large scale, for many people affected, and with little boundaries. 


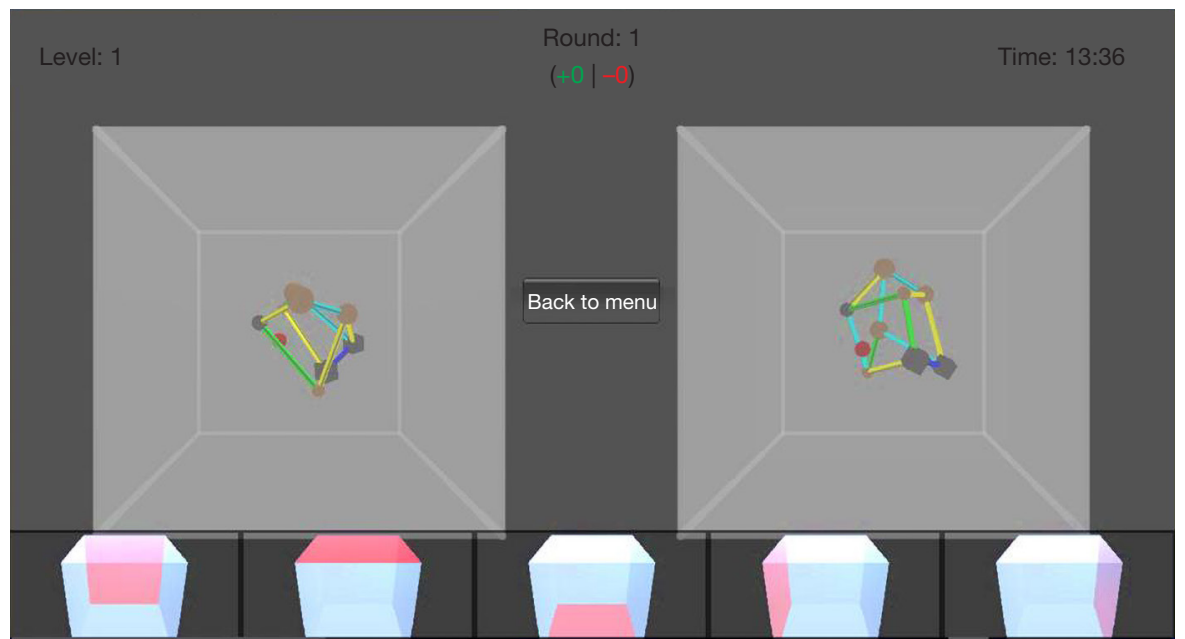

Figure 1 Screenshot of Mobilum. Left side of the split screen displays a cube containing a complex three-dimensional figure from the front. The task is to choose from five options (five boxes at the bottom of the screen) from which side the same figure is displayed on the right side of the split screen. In this example, participants see the figure from below on the right side. Hence, participants would have to touch the answer box in the middle out of the five options displayed at the bottom of the screen. If the right answer is chosen, the player gains a point, indicated by the green number after the plus on top of the screen and loses a point when a wrong answer is chosen, indicated by the red number after the minus on top of the screen.

\section{The development of Mobilum}

Mobilum was developed in our own lab by a team consisting of a professional game programmer (VM), game producer (RK) and methodological / design input by the other co-authors. The app was developed using a personal edition of the Unity 5 game engine (https://unity3d.com/ de/unity) and implemented in the programming language C\#. It is designed to run on Android mobile devices (e.g., smart phones, tablet computers). Major design specifications were ease of use, adequate engagement of mental rotation in order to solve the task, app stability, offline functionality and minimal hardware and operating system needs in order to run. The last aspect is of crucial importance as we intended the app to run on most mobile devices world-wide without trouble. In practice, only Android 2.3.3 (Gingerbread) as the operating system is needed allowing the app to run on relatively old devices. After implementing the changes that patients' feedback suggested (see below), the final version of Mobilum will be provided in the Google Play Store (https://play.google. com/store/apps) as an android app completely free of charge for research purposes. After downloading the app, it can be played offline on mobile devices.

Unfortunately, there is no option to save participants' scores as there is no data / participant management functionality. This means that researchers have to write down each participant's score, number of rounds played and of correct and wrong answers from the final screen of the app when the timer reached zero. As for now, there is no clear evidence that performance in a visuospatial task (e.g., score, time) is related to its effect to reduce intrusions, and in clinical contexts this game data is of no relevance. Still, we would like to include options to save the score for research purposes, since future studies might provide evidence for interesting correlations between game variables and outcome.

\section{The content of Mobilum}

In Mobilum, participants have to massively engage in mental rotation in order solve multiple three-dimensional tasks. Essentially, they have to "rotate" in an imaginative way around a virtual cube in order to decide from which perspective a complex geometrical figure is seen. Within a given time frame (adjustable between 1 and $30 \mathrm{~min}$ ) they have to solve as many tasks as possible (speed, plus score) and make as little mistakes as possible (precision, minus score). See Figure 1 and legend for illustrative instructions how the game is played. The main game screen is split 
in two halves. On the left side, a translucent cube is displayed that contains a complex three-dimensional figure, consisting of coloured three-dimensional geometric forms (e.g., spheres, cubes, tetrahedra) connected by coloured lines, always viewed from the front. On the right side of the split screen, the same figure is displayed from an alternative view. With front view always displayed on the left side, five other views are possible options on the right side of the split screen: back, top, bottom, left and right. Participants have to choose from those five options (displayed on the lower part of the screen) by touching the respective cube with the view highlighted. Immediately following the user's decision, the box on the bottom of the screen containing the correct answer is highlighted, and the cube on the right side of the screen slowly rotates to match the figure displayed on the left (as a visual feedback to graphically display the correct answer). If users correctly identify the viewing position, one point is awarded (green plus on top of the screen), if they are wrong, one point is subtracted (red minus). With more points gained, the difficulty level increases and figures inside the translucent cube become more complex. With decreasing scores (due to too complex figures), the difficulty level decreases correspondingly. This adaptive difficulty level system was implemented in order to always ensure a flowing gameplay experience with the task providing a challenge without being too frustrating. The timer (top right of screen) counts down from the initial time available for game play (adjustable between 1 and $30 \mathrm{~min}$ ) until it reaches zero and the game stops with the recent score being the final score. The final screen of the app then displays total score, number of rounds played and of correct and wrong answers. The goal is to achieve as many points as possible during the given time frame (speed) and make as little mistakes as possible (precision). The game play duration can be adjusted to suit different study design boundaries. In experiments in our lab, duration is typically set to 15 mins. Additionally, the difficulty level can also be changed in the options menu of the game (available to researchers, not participants). The suggested difficulty level is "adaptive" as described above. Researchers can still set the difficulty manually to a level between 1 and 10 (fixed), which are the levels the game chooses itself in "adaptive" mode according to participants' performance. With the difficulty level fixed at 1 , researchers can ensure that the game always remains easy, whereas they could set it to 10 in order to create a very challenging game with only the best participants reaching positive scores.

\section{First application in patients regarding usability and feasibility}

In order to conduct future clinical studies using Mobilum, a pilot study on usability and feasibility of the game was carried out. A total of $\mathrm{N}=16$ inpatient participants were included in the study, seven males and nine females. The participants were completing an inpatient psychosomatic and psychotherapeutic treatment with a duration of six to eight weeks. All participants were given extensive oral information about the pilot study, its purpose, scope, data handling and especially that they could stop testing the app at any time without reasons and that their participation would not interfere with their regular inpatient treatment. All patients provided oral consent to participate in the study. The mean age was 42.56 years with a standard deviation of 13.12, ranging from 23 to 65 years of age. All participants were of Caucasian origin. 14 participants were diagnosed with PTSD as the main diagnosis. Two female participants were diagnosed with a recurring major depression as the main diagnosis and did not suffer from PTSD. The participants were each given a 15 -min introduction to the Mobilum game in which they received instructions on the task, on the function of the app, and the tablet. They were asked to play Mobilum over a course of five consecutive days (Monday-Friday) and each day play the game for 15 mins. They were instructed to play on Level 1 on the first day and switch to Level "Adaptive" for the remaining four days, meaning that the difficulty of the task provided was changing depending on their performance. The participants were handed out the tablets each morning at 8:30 am and asked to return the tablets at $4: 30 \mathrm{pm}$. Within the given time frame, the participants were able to choose freely when to play the game in order to avoid an overlap with their regular treatment schedule. They were instructed to find a quiet space to play in order to avoid distractions competing for mental resources. At the end of the five-day period, an evaluation questionnaire was handed out containing 13 statements about satisfaction with the instructions provided, usability and feasibility. The subscale "instructions" included the following items: "The instructions were helpful", "The instructions were sufficient" and "The task was easy to understand". Regarding "usability", items included: "The design of the app is simple and effective", "I think most people could play this game", "Using the app was simple", "I could focus well on the task", "It was easy to change settings for the app", "I am satisfied with the operation of the app". Statements in the subscale "feasibility" included: 
"The mental rotation game was easy to complete", "My performance increased during the week", "I could easily adapt to the different Levels of difficulty", "I am satisfied with the feasibility of the game".

Also, they were asked to rate a statement saying "I could image using this app as part of my treatment over a longer period of time" and should afterwards indicate their overall satisfaction with the app ("Overall I am satisfied with the application”). The participants were asked to rank the statements on a five-point Likert scale ranging from completely disagree (I), partially disagree (II), neutral (III) partially agree (IV) to completely agree (V). Finally, the questionnaire contained an open comment section asking the participants to give personal feedback on the most positive and the most negative aspects of the game.

\section{Results of patients' feedback}

Subscores were created for "instruction", "usability", and "feasibility", and the overall mean score of all participants on all statements was computed. The "overall" mean was $\mathrm{M}=3.41, \mathrm{SD}=.74$ indicating that the mean answer was between "neutral" and "partially agree" with little deviation of the total scores from the mean. The subscale "instruction" showed a mean of $M=3.54, S D=1.06$. The mean for the subscale "usability" was $\mathrm{M}=3.81, \mathrm{SD}=1.78$ and for "feasibility" $M=3.17, \mathrm{SD}=0.83$. These results indicate a similar mean across all subscales with the highest mean score on usability and the lowest mean score on feasibility. The statement "I could image using this app as part of my treatment over a longer period of time" had an average of $M=2.86$ on the Likert scale with a standard deviation of $\mathrm{SD}=1.54$. The mean score for the statement "overall I am satisfied with the application" was $M=3.27, \mathrm{SD}=1.03$.

Overall, the results suggest that improvements need to be made for the Mobilum app due to the mean scores all centred around the middle of the five-point Likert scale. In order to get a more specific feedback, the open comment section was analysed. A total of 12 participants used the open comment section. Positive feedback was given in regard to: the change in difficulty according to the performance; a training effect ("i got better every day"); the statistics that were given (showing the amount of right and wrong answers); the simplicity of the application; the development of new mental strategies to change ones perspective and to solve the task; the concentration and focus on the task for a limited amount of time; the duration of the game (15 $\mathrm{min}$ as a perfect time frame); the immediately displayed solution of each new rotation task, once a decision was made. Moreover, participants indicated that the game required their concentration and that "ongoing negative thoughts" were absent during game play.

Negative feedback included that the fixation on the screen to observe two static figures (both translucent cubes) was difficult. Also, several participants replied that they soon felt frustrated, restless, that the mental rotation of static images was tiresome and that the game is mostly suited for people with mental rotation abilities.

In regard to usability and design, four participants stated that the contrast of colours in the app was insufficient. In line with this, a lighter background and brighter colours were suggested as well as better visibility of the menu function (letter size, colour and font). Also, the automatic rotation of the right cube as soon as a choice is made during the game in order to show the right answer was perceived as too fast and the time to process any mistake until the next task appeared was perceived as too short. Overall, several participants indicated that the task was too difficult.

In sum, it can be concluded that changes have to be made in order to make the application more appealing, increase the learning effect and increase acceptance, motivation and concentration. Colours and contrasts need to be intensified, the difficulty of the starting level has to be decreased in order to avoid frustration in the beginning, the menu section should be clearly readable and understandable and the rotation speed of the cubes should be slowed in order to increase a learning effect (where did I go wrong?). The changes would not interfere with the core task of the app (mental rotation).

\section{Conclusions}

Many studies using Tetris have shown that a visuospatial task can reduce the frequency of intrusive visual memories in healthy as well as patient populations. Scientific and commercial issues have driven our effort to develop a new visuospatial task, Mobilum, as an alternative to further explore applications in participants in future studies. Mobilum is a task that massively engages mental rotation as participants have to "rotate" around a translucent virtual cube in order to decide from which perspective a complex geometrical figure is seen. First application of Mobilum in $\mathrm{N}=16$ patients yielded mixed feedback. On one hand, the design specification was met as the app itself is easy to use and the task is actually engaging and needs mental rotation and concentration to be solved. Additionally, the feedback that "ongoing negative thoughts" were less during game 
play hints at potential therapeutic effects of the game. On the other hand, there were many negative points regarding particular issues of the game (mainly regarding appearance of the app). The most important feedback, though, was that the difficulty level of the task was mostly too high. This caused frustration for some participants and was considered stressful and tiring. Consequently, small appearance issues and foremost the overall difficulty level have to be changed before using Mobilum in future studies. It is of key importance that the first difficulty levels are simpler in order to increase initial acceptance of the game and avoid frustration.

\section{Next steps}

The app Mobilum has to be improved, which foremost regards changes in difficulty level and the graphical appearance. Afterwards it can be uploaded to the Google Play Store to be used other research groups. Then, future studies, including those in our own lab, could proceed in a similar manner as previous studies using Tetris: First, healthy participants should view a trauma film and play various interventions afterwards (either directly, 24 or 72 $\mathrm{h}$ later) to observe the effect on the number of intrusions in the week following the film. In those studies, Tetris and Mobilum should be compared directly against each other with all other variables kept constant. This way, it could be established that Mobilum is in principle as effective as Tetris. Further studies should then be conducted in populations suffering from (or at high risk for) PTSD. Patients and/or risk populations could either play Tetris or Mobilum after being reminded of a traumatic scene in order to specifically reduce the frequency of intrusive memories for that particular scene. Positive results for latter populations would be a key step in providing Mobilum as a freely available and easy-to-use tool for the specific reduction of intrusive memories.

\section{Acknowledgments}

The companies Daedalic (RK) and Sunlab (VM) were not involved in the development of Mobilum. RK and VM developed the app in their free time.

\section{Footnote}

Conflicts of Interest: The authors have no conflicts of interest to declare.

Ethical Statement: The authors are accountable for all aspects of the work in ensuring that questions related to the accuracy or integrity of any part of the work are appropriately investigated and resolved. Oral informed consent was obtained from the patient for publication of this manuscript.

\section{References}

1. World Health Organization. Guidelines for the management of conditions specifically related to stress. Geneva: World Health Organization, 2013.

2. Atwoli L, Stein DJ, Koenen KC, et al. Epidemiology of posttraumatic stress disorder: prevalence, correlates and consequences. Curr Opin Psychiatry 2015;28:307-11.

3. National Institute for Health and Care Excellence. NICE Guideline NG116: Post-traumatic stress disorder, 2018. Available online: http://www.nice.org.uk/guidance/ng116

4. Schäfer I, Gast U, Hofmann A, et al. S3 - Leitlinie Posttraumatische Belastungsstörung. Trauma \& Gewalt, 2011.

5. American Psychological Association. Clinical Practice Guideline for the Treatment of Posttraumatic Stress Disorder (PTSD), 2017. Available online: http://www.apa. org/ptsd-guideline

6. Bisson JI, Roberts NP, Andrew M, et al. Psychological therapies for chronic post-traumatic stress disorder (PTSD) in adults. Cochrane Database of Systematic Reviews 2013. DOI: 10.1002/14651858.CD003388.pub4.

7. Kessler RC. Posttraumatic stress disorder: the burden to the individual and to society. J Clin Psychiatry 2000;61 Suppl 5:4-12; discussion 13-4.

8. Holmes EA, Ghaderi A, Harmer CJ, et al. The Lancet Psychiatry Commission on psychological treatments research in tomorrow's science. Lancet Psychiatry 2018;5:237-86.

9. Woud ML, Holmes EA, Postma P, et al. Ameliorating intrusive memories of distressing experiences using computerized reappraisal training. Emotion 2012;12:778-84.

10. Woud ML, Postma P, Holmes EA, et al. Reducing analogue trauma symptoms by computerized reappraisal training - considering a cognitive prophylaxis? J Behav Ther Exp Psychiatry 2013;44:312-5.

11. Woud ML, Verwoerd J, Krans J. Modification of cognitive biases related to posttraumatic stress: A systematic review and research agenda. Clin Psychol Rev 2017;54:81-95.

12. Woud ML, Blackwell SE, Cwik JC, et al. Augmenting inpatient treatment for post-traumatic stress disorder with a computerised cognitive bias modification procedure targeting appraisals (CBM-App): protocol for a randomised 
controlled trial. BMJ Open 2018;8:e019964.

13. Bomyea J, Stein MB, Lang AJ. Interference control training for PTSD: A randomized controlled trial of a novel computer-based intervention. Journal of Anxiety Disorders 2015;34:33-42.

14. Woud ML, Heeren A, Shkreli L, et al. Investigating the effect of proactive interference control training on intrusive memories. Eur J Psychotraumatol 2019;10:1611092.

15. Brewin CR. A cognitive neuroscience account of posttraumatic stress disorder and its treatment. Behav Res Ther 2001;39:373-93.

16. Ehlers A, Clark DM. A cognitive model of posttraumatic stress disorder. Behav Res Ther 2000;38:319-45.

17. Andrade J, Kavanagh D, Baddeley A. Eye-movements and visual imagery: a working memory approach to the treatment of post-traumatic stress disorder. Br J Clin Psychol 1997;36:209-23.

18. Baddeley AD, Andrade J. Working memory and the vividness of imagery. J Exp Psychol Gen 2000;129:126-45.

19. van den Hout M, Muris P, Salemink E, et al. Autobiographical memories become less vivid and emotional after eye movements. Br J Clin Psychol 2001;40:121-30.

20. Engelhard IM, van Uijen SL, van den Hout MA, et al. The impact of taxing working memory on negative and positive memories. Eur J Psychotraumatol 2010. doi: 10.3402/ejpt. v1i0.5623

21. Engelhard IM, van den Hout MA, Smeets MA. Taxing working memory reduces vividness and emotional intensity of images about the Queen's Day tragedy. J Behav Ther Exp Psychiatry 2011;42:32-7.

22. Shapiro F. Eye movement desensitization and reprocessing (EMDR): Basic principles, protocols, and procedures. 2nd ed. New York: Guilford Press, 2001.

23. Lee CW, Cuijpers P. A meta-analysis of the contribution of eye movements in processing emotional memories. J Behav Ther Exp Psychiatry 2013;44:231-9.

24. Holmes EA, James EL, Coode-Bate T, et al. Can playing the computer game "Tetris" reduce the build-up of flashbacks for trauma? A proposal from cognitive science. PLoS One 2009;4:e4153.

25. Holmes EA, James EL, Kilford EJ, et al. Key steps in developing a cognitive vaccine against traumatic flashbacks: visuospatial Tetris versus verbal Pub Quiz. PLoS One 2010;5:e13706.

26. Deeprose C, Zhang S, Dejong H, et al. Imagery in the aftermath of viewing a traumatic film: using cognitive tasks to modulate the development of involuntary memory. J Behav Ther Exp Psychiatry 2012;43:758-64.
27. Lau-Zhu A, Holmes EA, Butterfield S, et al. Selective Association Between Tetris Game Play and Visuospatial Working Memory: A Preliminary Investigation. Appl Cogn Psychol 2017;31:438-45.

28. Iyadurai L, Blackwell SE, Meiser-Stedman R, et al. Preventing intrusive memories after trauma via a brief intervention involving Tetris computer game play in the emergency department: a proof-of-concept randomized controlled trial. Molecular Psychiatry 2018;23:674-82.

29. Horsch A, Vial Y, Favrod C, et al. Reducing intrusive traumatic memories after emergency caesarean section: A proof-of-principle randomized controlled study. Behav Res Ther 2017;94:36-47.

30. Misanin JR, Miller RR, Lewis DJ. Retrograde amnesia produced by electroconvulsive shock after reactivation of a consolidated memory trace. Science 1968;160:554-5.

31. Nader K, Schafe GE, LeDoux JE. The labile nature of consolidation theory. Nat Rev Neurosci 2000;1:216-9.

32. Monfils MH, Holmes EA. Memory boundaries: opening a window inspired by reconsolidation to treat anxiety, trauma-related, and addiction disorders. The Lancet Psychiatry 2018;5:1032-42.

33. James EL, Bonsall MB, Hoppitt L, et al. Computer Game Play Reduces Intrusive Memories of Experimental Trauma via Reconsolidation-Update Mechanisms. Psychol Sci 2015;26:1201-15.

34. Hagenaars MA, Holmes EA, Klaassen F, et al. Tetris and Word games lead to fewer intrusive memories when applied several days after analogue trauma. Eur J Psychotraumatol 2017;8:1386959.

35. Kessler H, Schmidt AC, James EL, et al. Visuospatial computer game play after memory reminder delivered three days after a traumatic film reduces the number of intrusive memories of the experimental trauma. J Behav Ther Exp Psychiatry 2019. doi: 10.1016/ j.jbtep.2019.01.006.

36. Kessler H, Holmes EA, Blackwell SE, et al. Reducing intrusive memories of trauma using a visuospatial interference intervention with inpatients with posttraumatic stress disorder (PTSD). J Consult Clin Psychol 2018;86:1076-90.

doi: $10.21037 /$ mhealth.2019.09.15

Cite this article as: Kessler H, Dangellia L, Kessler R, Mahnke V, Herpertz S, Kehyayan A. Mobilum—a new mobile app to engage visuospatial processing for the reduction of intrusive visual memories. mHealth 2019;5:49. 